\title{
Antimicrobial activity of Lactobacillus plantarum strains against Salmonella pathogens
}

\author{
Desislava Teneva ${ }^{1}$, Rositsa Denkova ${ }^{2}$, Bogdan Goranov ${ }^{3}$, \\ Zapryana Denkova ${ }^{1}$, Georgi Kostov ${ }^{4}$ \\ 1 - University of Food Technologies, Department of Microbiology, Plovdiv, Bulgaria \\ 2 - University of Food Technologies, Department of Biochemistry and molecular biology, \\ Plovdiv, Bulgaria \\ 3 - LBLact, Plovdiv, Bulgaria \\ 4 - University of Food Technologies, Department of Wine and brewing, Plovdiv, Bulgaria
}

\section{Keywords: \\ Probiotic \\ Lactobacillus \\ Pathogen \\ Antimicrobial \\ Co-culturing \\ Salmonella}

Article history:

Received

28.01.2017

Received in revised form 22.03.2017

Accepted 30.03.2017

\section{Corresponding}

author:

Rositsa Denkova

E-mail:

rositsa_denkova@ mail.bg

DOI:

10.24263/2304-

974X-2017-6-1-14

\section{Abstract}

Introduction. Lactic acid bacteria produce various compounds such as organic acids, diacetyl, hydrogen peroxide and bacteriocin during lactic fermentations. All of these can antagonize the growth of some spoilage and pathogenic bacteria in foods.

Materials and methods. To determine the antimicrobial activity of Lactobacillus plantarum D1 and Lactobacillus plantarum D2 against Salmonella sp. and Salmonella abony ATCC 6017, the method of co-culturing was applied. The study was conducted under static conditions at $37 \pm 1{ }^{\circ} \mathrm{C}$ for 72 hours, taking samples at $0,12,24,36,48,60$ and $72 \mathrm{~h}$ and monitoring the changes in the titratable acidity and the concentration of viable cells of both the pathogens and the Lactobacillus plantarum strains.

Results and discussion. In the single-strain cultivation of each Lactobacillus plantarum strain and each Salmonella strain high concentration of viable cells were achieved by the $24^{\text {th }}$ hour and it was maintained by the end of the culturing. In the co-culturing of each Lactobacillus plantarum strain and each Salmonella strain, the Lactobacillus strain was not significantly influenced by the presence of any of the Salmonella strains. But the number of viable cells of the pathogens was greatly reduced, the reduction being strainspecific. In the co-culturing of each Lactobacillus plantarum strain and Salmonella abony ATCC 6017, the concentration of viable cells of the pathogen strain was reduced by the $60^{\text {th }} \mathrm{h}$. In the co-culturing of each Lactobacillus plantarum strain and Salmonella sp., the concentration of viable cells of the pathogen strain was reduced by the $72^{\text {th }} \mathrm{h}$. The observed antimicrobial activity was due to a great extent to the acidification of the medium because of the production and accumulation of lactic and other oraganic acids.

Conclusions. The demonstrated antimicrobial activity is a prerequisite for further research on the probiotic potential of the two Lactobacillus plantarum strains for their inclusion in the composition of probiotic preparations and starters for probiotic functional foods. 


\section{Introduction}

Lactic acid bacteria play an important role in food fermentation processes. Raw foods such as milk, fruits, vegetables or meat are often preserved by lactic acid fermentation. These organisms produce various compounds such as organic acids, diacetyl, hydrogen peroxide and bacteriocin during lactic fermentations. All of these can antagonize the growth of some spoilage and pathogenic bacteria in foods $[1,2]$.

Most of the probiotic lactobacilli in human foods are supplied in highly concentrated forms containing more than $10^{10} \mathrm{cfu} / \mathrm{cm}^{3}$. The ability of Lactobacillus strains to adhere to the mucosal surfaces of the intestine and the subsequent long or short term colonization has long been one of the most commonly encountered criteria for the selection of probiotic strains $[3,4,5]$.

Enteric disorders are one of the most important problems in the food industry, with salmonellosis and colibacillosis regarded as the major bacterial diseases occurring in human. Salmonella and Escherichia coli infections range from severe acute disease to mild infections of a chronic nature [6].

The purpose of the present work was to study the antimicrobial activity of Lactobacillus plantarum D1 and Lactobacillus plantarum D2 against the Gram-positive pathogenic microorganisms Salmonella sp. (clinical isolate) and Salmonella abony ATCC 6017, that cause toxicoses and toxicoinfections.

\section{Materials and methods}

\section{Microorganisms}

Lactobacillus plantarum D1 and Lactobacillus plantarum D2, isolated from salad dressings;

test pathogenic microorganisms Salmonella sp. (clinical isolate) and Salmonella abony ATCC 6017.

\section{Media}

\subsection{MRS - broth medium}

Composition $\left(\mathrm{g} / \mathrm{dm}^{3}\right)$ : peptone from casein -10 ; yeast extract -4 ; meat extract -8 ; glucose $-20 ; \mathrm{K}_{2} \mathrm{HPO}_{4}-2$; sodium acetate -5 ; diammonium citrate $-2 ; \mathrm{MgSO}_{4}-0.2$; $\mathrm{MnSO}_{4}-0.04$; Tween $80-1 \mathrm{~cm}^{3} / \mathrm{dm}^{3} ; \mathrm{pH}=6.5$. Sterilization -15 minutes at $118^{\circ} \mathrm{C}$.

\subsection{LAPTg10 - agar medium}

Composition $\left(\mathrm{g} / \mathrm{dm}^{3}\right)$ : peptone -15 ; yeast extract -10 ; tryptone -10 ; glucose -10 ; Tween $80-1 \mathrm{~cm}^{3} / \mathrm{dm}^{3}$, agar $-15 . \mathrm{pH}=6.6-6.8$. Sterilization -20 minutes at $121^{\circ} \mathrm{C}$.

2.3. LBG - agar medium

Composition $\left(\mathrm{g} / \mathrm{dm}^{3}\right)$ : tryptone -10 ; yeast extract $-5 ; \mathrm{NaCl}-10$; glucose -10 ; agar $-15 ; \mathrm{pH}=7,5$. Sterilization -20 minutes at $121^{\circ} \mathrm{C}$.

\section{Determination of the antimicrobial activity against pathogenic microorganisms - by co-culturing}

To determine the antimicrobial activity of the studied lactobacilli strains against the two pathogens a 48 hour cultural suspension of each Lactobacillus plantarum strain was used. Separate cultivation of the two Lactobacillus plantarum and the two Salmonella strains as well as co-culturing of each of the two Lactobacillus plantarum strains and each Salmonella strain included in the study were conducted. For the examination of the coculturing, $0.5 \mathrm{~cm}^{3}$ of the suspension of the Lactobacillus plantarum strain, $0.5 \mathrm{~cm}^{3}$ of the 
suspension of the Salmonella strain and $9 \mathrm{~cm}^{3}$ of culture medium (MRS-broth medium) were mixed. In the control of each Lactobacillus plantarum strain and in the control of each pathogen, $9.5 \mathrm{~cm}^{3}$ of the MRS-broth medium were mixed with $0.5 \mathrm{~cm}^{3}$ of the suspension of the Lactobacillus plantarum strain or of the suspension of the Salmonella strain, respectively. The study was conducted under static conditions in a thermostat at $37 \pm 1^{\circ} \mathrm{C}$ for 72 hours, taking samples at $0,12,24,36,48,60$ and $72 \mathrm{~h}$ and monitoring the changes in the titratable acidity and the concentration of viable cells of both the pathogens and the Lactobacillus plantarum strains. The determination of the number of viable cells was done by the spread plate method on LAPTg10-agar (for the enumeration of lactobacilli), on LBG-agar (for the enumeration of pathogens). The titratable acidity was determined according to a standard protocol [7].

\section{Results and discussion}

In the study of the antimicrobial activity of the two Lactobacillus plantarum against the two Salmonella strains by the method of co-culturing, the dynamics of the change in the number of viable cells of both the lactobacilli and the pathogens and in the titratable acidity were monitored (Fig. 1, Fig. 2, Fig. 3, Fig. 4, Fig. 5, Fig. 6, Fig. 7 and Fig. 8).

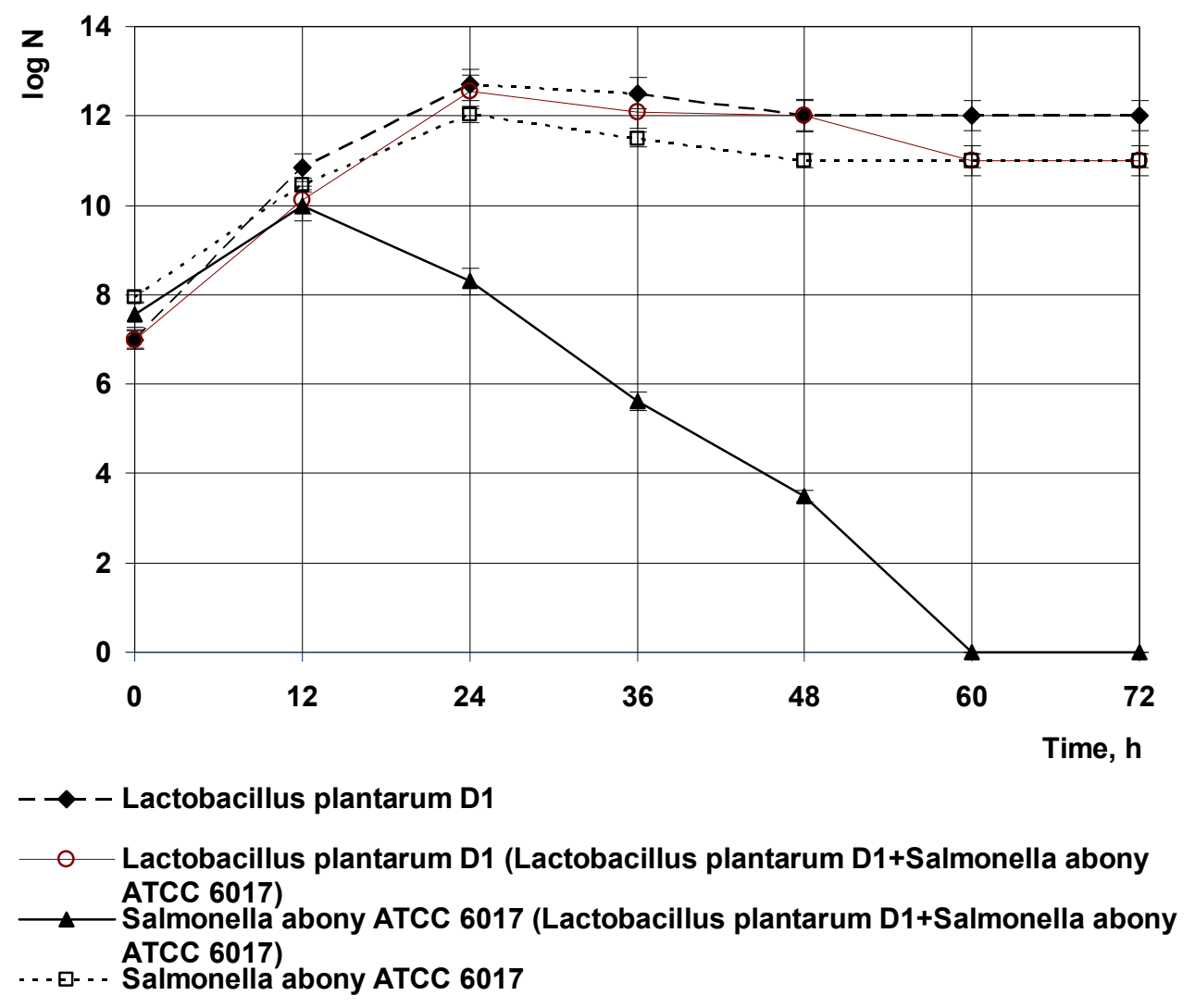

Figure 1. Changes in the number of viable calls of Lactobacillus plantarum D1 and Salmonella abony ATCC 6017 in single-strain culturing and in a mixed population at $37 \pm 1^{\circ} \mathrm{C}$ 
In the single-strain cultivation of each Lactobacillus plantarum strain and each Salmonella strain high concentration of viable cells were achieved by the $24^{\text {th }}$ hour and it was maintained by the end of the culturing. In the co-culturing of each Lactobacillus plantarum strain and each Salmonella strain, the Lactobacillus strain was not significantly influenced by the presence of any of the Salmonella strains. But the number of viable cells of the pathogens was greatly reduced, the reduction being strain-specific. The obtained results were commesurable with the observations described in [9].

The Lactobacillus plantarum and Salmonella strains entered the stationary growth phase at the $24^{\text {th }} \mathrm{h}$, reaching maximum concentration of viable cells - above $10^{12} \mathrm{cfu} / \mathrm{cm}^{3}$ (Fig. 1, Fig. 2, Fig. 5 and Fig. 6). Meanwhile the titratable acidity of the medium in the culturing of both the two strains of lactobacilli reached $140^{\circ} \mathrm{T}$ (Fig. 3, Fig. 4, Fig. 7 and Fig. 8). A similar trend was established in the single-strain culture of the two Salmonella strains. Therefore, Salmonella sp. Entered the stationary growth phase at the $12^{\text {th }} \mathrm{h}$, while Salmonella abony ATCC 6017 entered it at the $24^{\text {th }} \mathrm{h}$, reaching concentrations of viable cells about $10^{12} \mathrm{cfu} / \mathrm{cm}^{3}$ (Fig. 1, Fig. 2, Fig. 5 and Fig. 6).

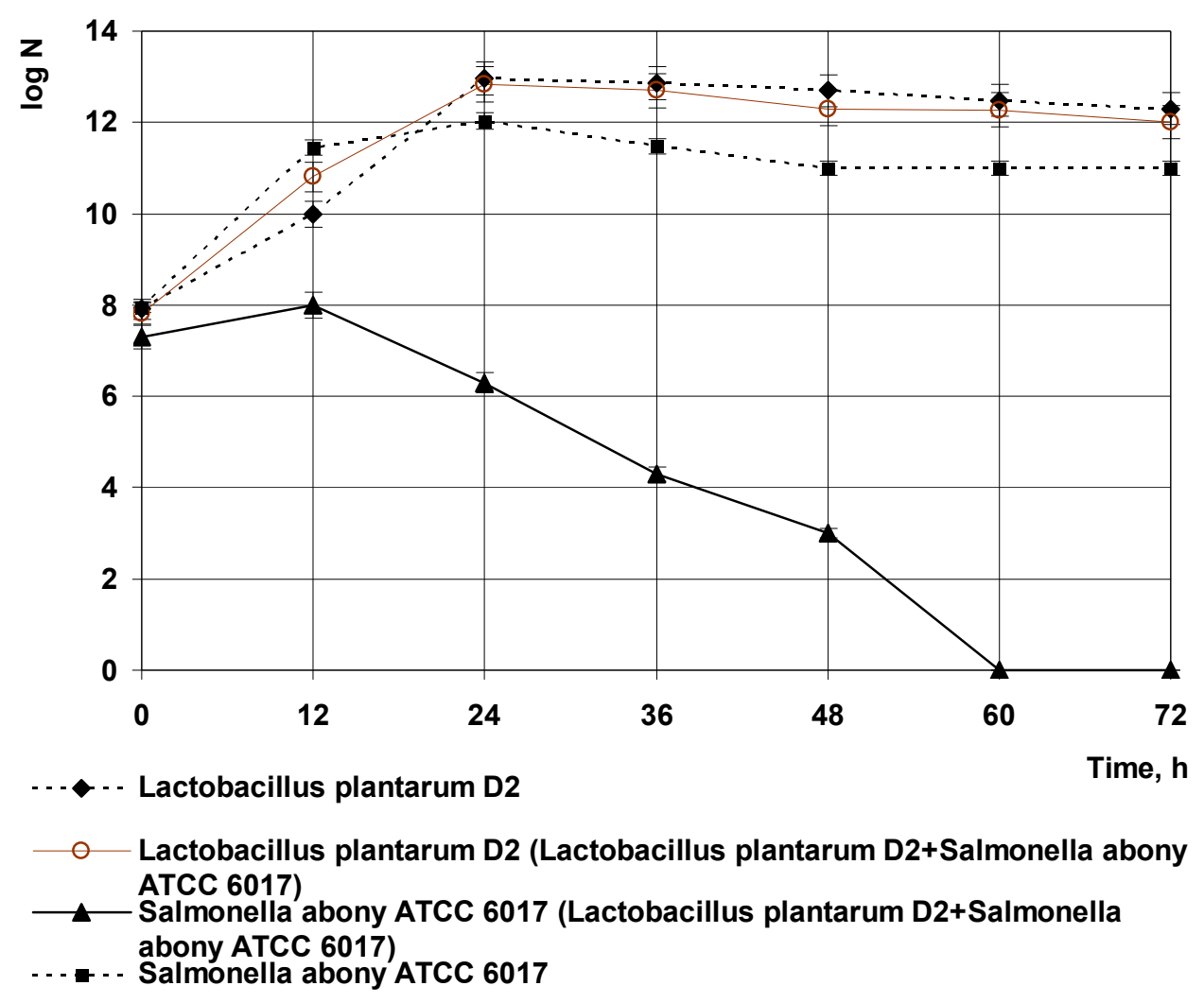

Figure 2. Changes in the number of viable calls of Lactobacillus plantarum D2 and Salmonella abony ATCC 6017 in single-strain culturing and in a mixed population at $37 \pm 1^{\circ} \mathrm{C}$. 
In the co-culturing of each Lactobacillus plantarum strain and Salmonella abony ATCC 6017 , the concentration of viable cells of the pathogen strain was reduced by the $60^{\text {th }}$ h (Fig. 3 and Fig. 4).

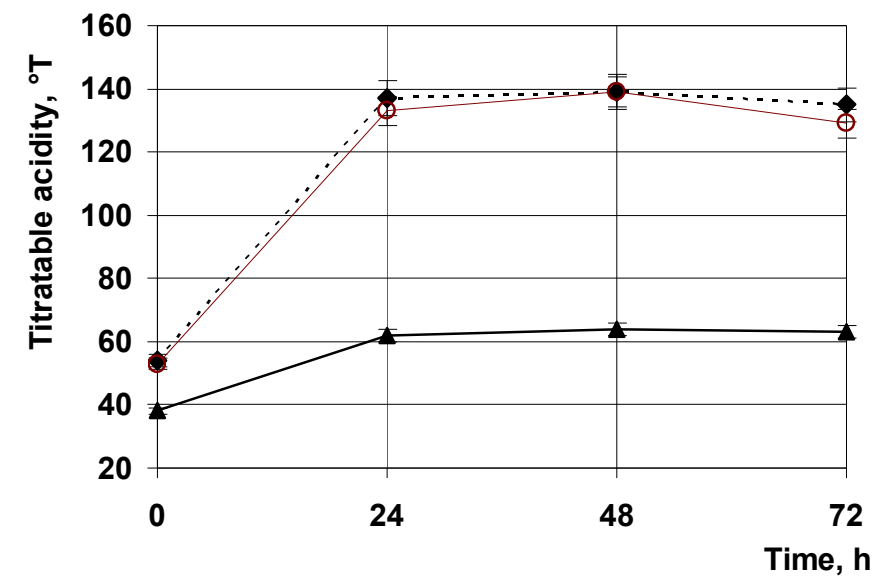

.... Lactobacillus plantarum D1

- Lactobacillus plantarum D1 + Salmonella abony ATCC 6017

$\_$Salmonella abony ATCC 6017

Figure 3. Changes in the titratable acidity of the medium in single-strain culturing and in a mixed population of Lactobacillus plantarum D1 and Salmonella abony ATCC 6017 at $37 \pm 1{ }^{\circ} \mathrm{C}$.

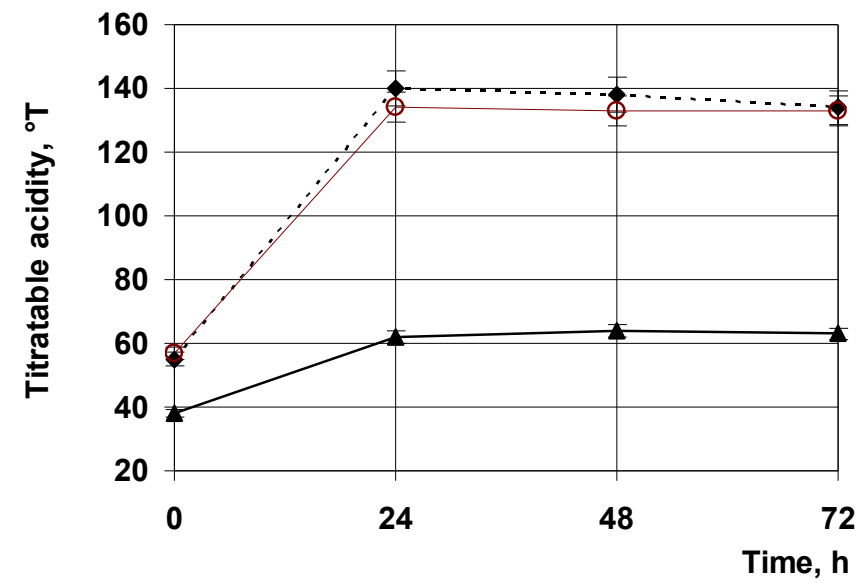

..... Lactobacillus plantarum D2

- Lactobacillus plantarum D2 + Salmonella abony ATCC 6017

$\_$Salmonella abony ATCC 6017

Figure 4. Changes in the titratable acidity of the medium in single-strain culturing and in a mixed population of Lactobacillus plantarum D2 and Salmonella abony ATCC 6017 at $37 \pm 1{ }^{\circ} \mathrm{C}$ 


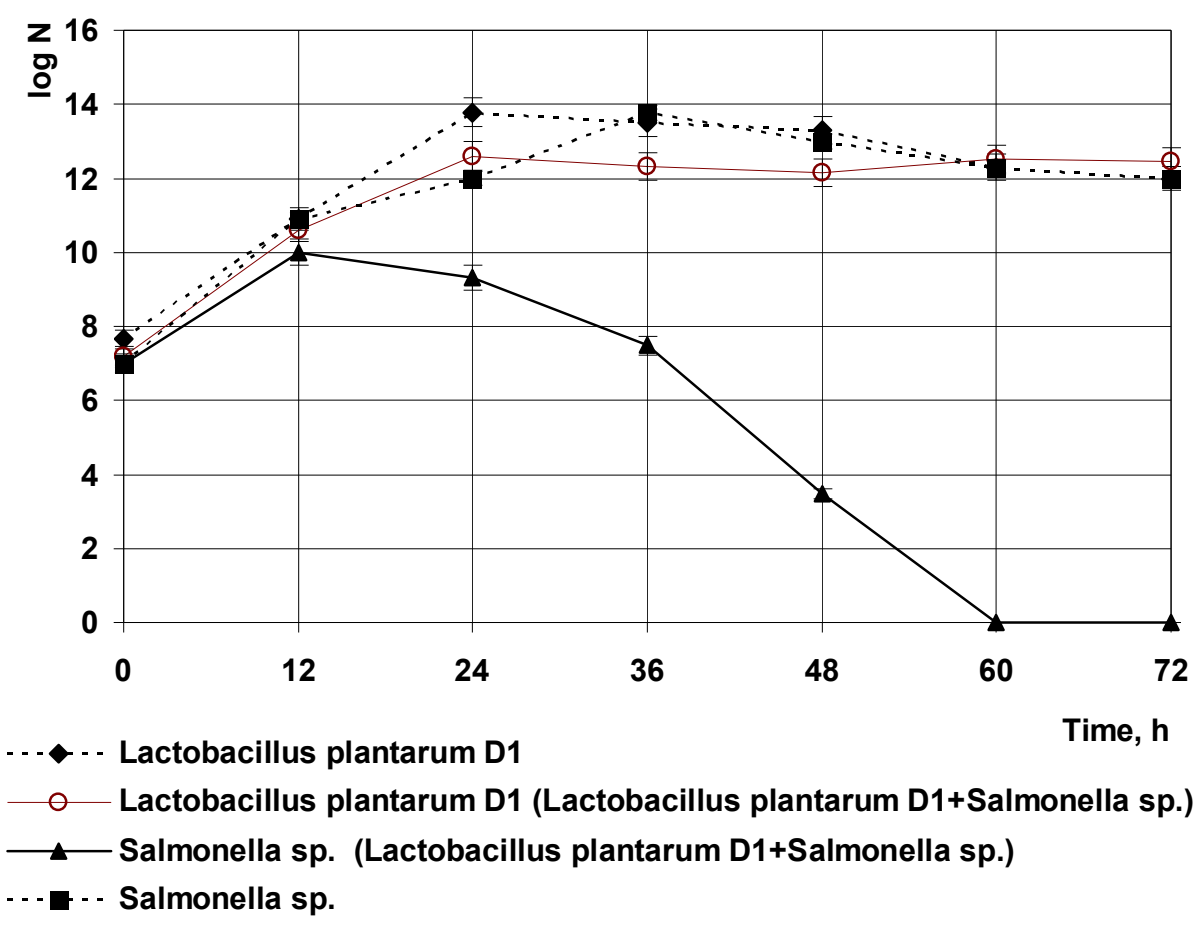

Figure 5. Changes in the number of viable calls of Lactobacillus plantarum D1 and Salmonella sp. In single-strain culturing and in a mixed population at $37 \pm 1{ }^{\circ} \mathrm{C}$.

In the co-culturing of each Lactobacillus plantarum strain and Salmonella sp., the concentration of viable cells of the pathogen strain was reduced by the $72^{\text {th }} \mathrm{h}$ (Fig. 7 and Fig. 8). The two Salmonella strains differed in their growth characteristics. In the coculturing of the two Salmonella strains with each of the two Lactobacillus plantarum strains, a slight increase in the number of living cells was observed, but it had different behavior depending on the very Lactobacillus plantarum strain.

The observed antimicrobial activity of the two Lactobacillus plantarum strains included in the present study was due to the production and accumulation of lactic and other organic acids. According to Helander et al. [8], L. plantarum produces a variety of low molecular mass compounds including acids, alcohols, carbon dioxide, diacetyl, hydrogen peroxide and other metabolites. Many of these metabolites have a broad activity spectrum against other species, and their production is largely affected by the food matrix itself. [6].

The L. plantarum strains isolated from salad dressings reduced the amount of viable cells of the two Salmonella strains in a mixed population (Lactobacillus plantarum and Salmonella strain) in the present in vitro study. The obtained results confirm the research by Denkova R. et al., 2013 [9]. But Lactobacillus plantarum D1 and Lactobacillus plantarum D2 reduced the number of living cells of Salmonella abony NTCC 6017 by the $60^{\text {th }} \mathrm{h}$, while Lactobacillus plantarum X2 (isolated from spontaneously fermented sourdough) could not do so even by the $72^{\text {nd }} \mathrm{h}$. On the other hand, Lactobacillus plantarum F3 (isolated from spontaneously fermented sourdough) and Lactobacillus plantarum LBRZ12 reduced the 
Salmonella abony NTCC 6017 living cells by the $60^{\text {th }} \mathrm{h}$, in compliance with the results reported in the present manuscript. Hence, Lactobacillus plantarum D1 and Lactobacillus plantarum D2 possess higher antimicrobial activity against Salmonella abony NTCC 6017 than Lactobacillus plantarum X2 [9]. Lactobacillus plantarum F3 (isolated from spontaneously fermented sourdough) managed to supress all Salmonella sp. (clinical isolate) cells by the $72^{\text {nd }} \mathrm{h}$, which is in compliance with the obtained results for the same pathogen in its co-culturing with Lactobacillus plantarum D1 and Lactobacillus plantarum D2. But Lactobacillus plantarum X2 and Lactobacillus plantarum LBRZ12 demonstrated lower antimicrobial activity against Salmonella sp. (clinical isolate) - the number of viable pathogen cells by the $72^{\text {nd }} \mathrm{h}$ was $10^{3} \mathrm{cfu} / \mathrm{cm}^{3}$ [9]. After additional research on the probiotic properties of the two Lactobacillus plantarum strains, they can be included in the composition of probiotic preparations and starters for functional probiotic foods and beverages. This in turn would ensure the microbiological safety of the foods and beverages. Moreover, upon intake the high concentration of viable cells of lactobacilli will provide the necessary beneficial flora to maintain the balance in the gastrointestinal tract and perform its inherent preventive role.

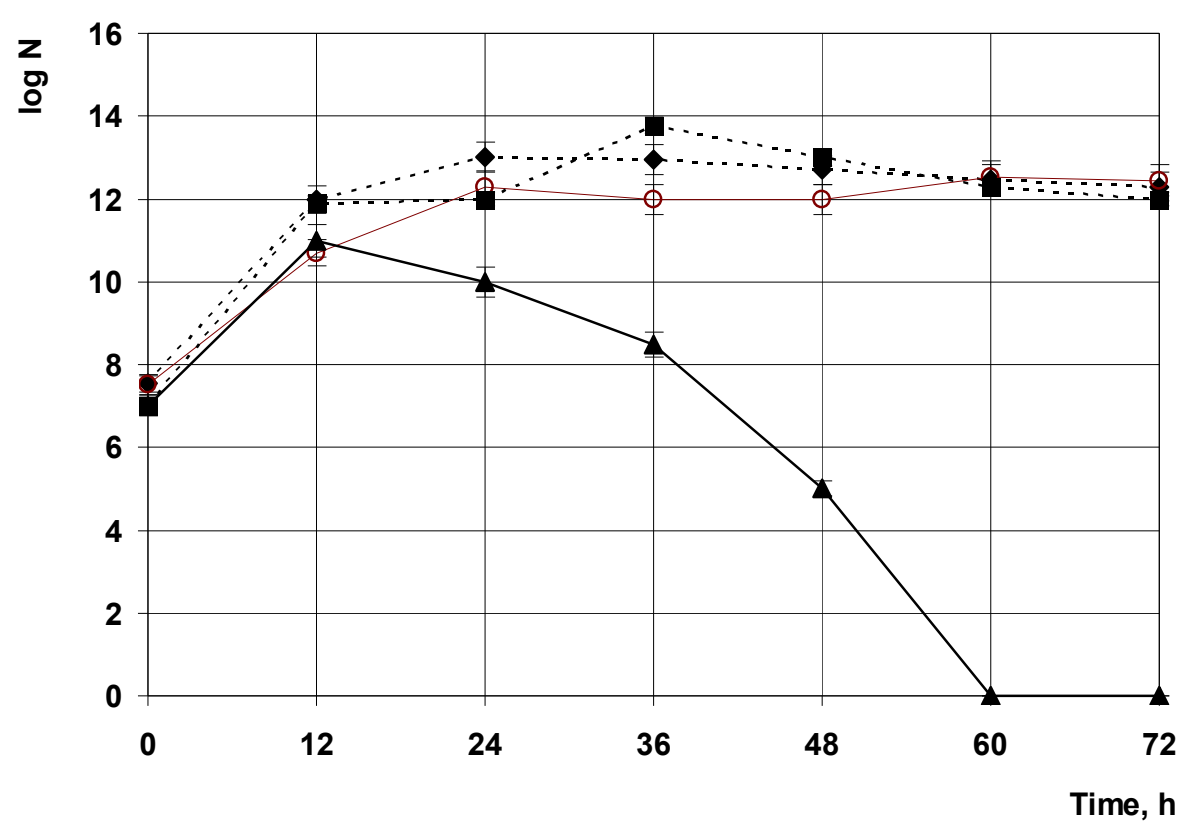

.... Lactobacillus plantarum D2

- L Lactobacillus plantarum D2 (Lactobacillus plantarum D2+Salmonella sp.)

$\longleftarrow$ Salmonella sp. (Lactobacillus plantarum D2+Salmonella sp.)

-... - Salmonella so.

Figure 6. Changes in the number of viable calls of Lactobacillus plantarum D2 and Salmonella sp. In single-strain culturing and in a mixed population at $37 \pm 1^{\circ} \mathrm{C}$. 


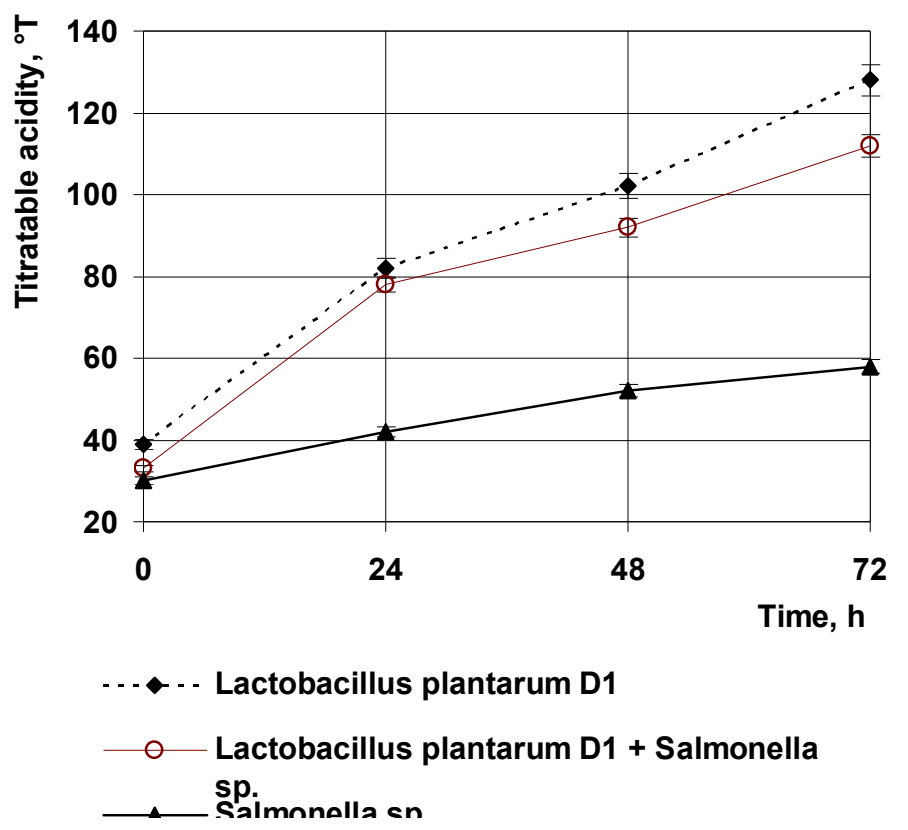

Figure 7. Changes in the titratable acidity of the medium in single-strain culturing and in a mixed population of Lactobacillus plantarum D1 and Salmonella sp. at $37 \pm 1{ }^{\circ} \mathrm{C}$.

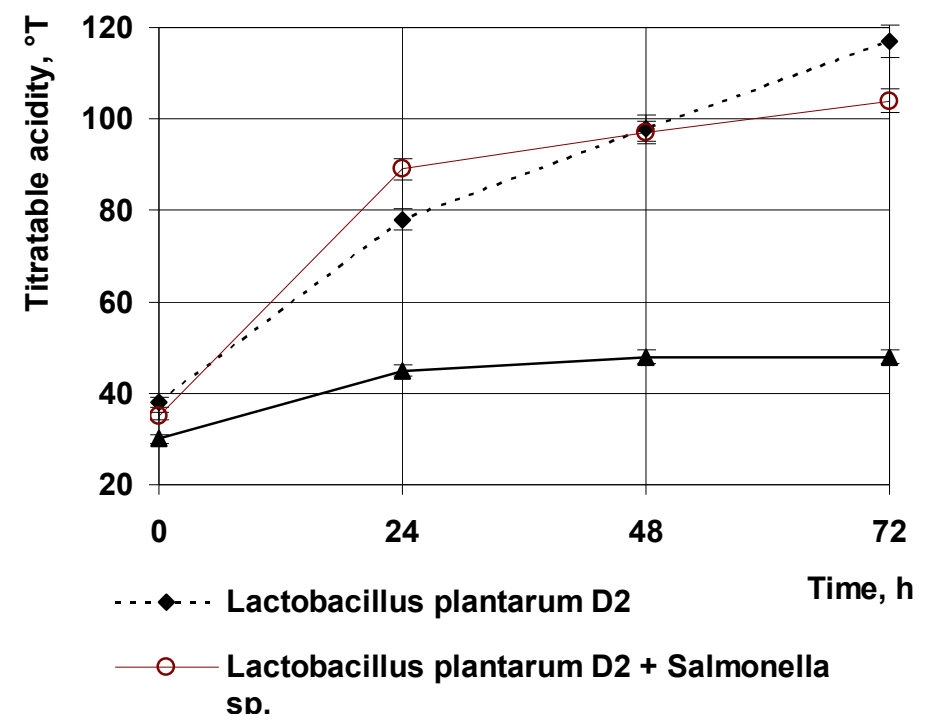

Figure 8. Changes in the titratable acidity of the medium in single-strain culturing and in a mixed population of Lactobacillus plantarum D2 and Salmonella sp. At $37 \pm 1^{\circ} \mathrm{C}$. 


\section{Conclusion}

Lactobacillus plantarum D1 and Lactobacillus plantarum D2 maintained high concentrations of viable cells in single-strain culturing and in co-culture at a temperature of $37 \pm 1^{\circ} \mathrm{C}$. Both Lactobacillus plantarum strains inhibited significantly the growth of the two Salmonella pathogens. The observed antimicrobial activity was due to a great extent to the acidification of the medium because of the production and accumulation of lactic and other oraganic acids. The demonstrated antimicrobial activity is a prerequisite for further research on the probiotic potential of the two Lactobacillus plantarum strains for their inclusion in the composition of probiotic preparations and starters for probiotic functional foods.

\section{References}

1. Abdelbasset M., Djamila K. (2008), Antimicrobial Activity of Autochthonous Lactic Acid Bacteria Isolated From Algerian Traditional Fermented Milk, Raib. Afr. J. Biotechnol., 7, pp. 2908-2914.

2. Oyetayo V.O., Adetuyi F.C., Akinyosoye F.A. (2003), Safety And Protective Effect of Lactobacillus acidophilus and Lactobacillus casei Used as Probiotic Agent in vivo, Afr. J. Biotech., 2, pp. 448-452.

3. Collado M.C., Gueimonde M., Hernandez M., Sanz Y., Salminen S. (2005), Adhesion of Selected Bifidobacterium Strains to Human Intestinal Mucus and Its Role in Enteropathogen Exclusion, J. Food Prot., 68, pp. 2672-2680.

4. Darsanaki R.K., Rokhi M., Aliabadi M., Issazadeh K. (2012), Antimicrobial Activities of Lactobacillus Strains Isolated From Fresh Vegetables, Middle-East Journal of Scientific Research, 11 (9), pp. 1216-1219.

5. Maragkoudakis P.A., Zoumpopoulou G., Miaris C., Kalantzopoulos G., Pot B., Tsakalidou E. (2006), Probiotic Potential of Lactobacillus Strains Isolated From Dairy Products, International Dairy Journal, 16, pp. 189-199.

6. Dec M., Puchalski A., Nowaczek A., Wernicki A. (2016), Antimicrobial Activity of Lactobacillus Strains of Chicken Origin Against Bacterial Pathogens, Int. Microbiol., 19, pp. 57-67.

7. Denkova Z. (2005), Production and application of probiotics, DSc thesis. Plovdiv, Bulgaria.

8. Helander I. M., von Wright A., Mattila Sandholm T-M. (1999), Trends in Food Science \& Technology, 8(5), pp. 146-150.

9. Denkova R., Denkova Z., Yanakieva V., Blazheva D. (2013), Antimicrobial activity of probiotic lactobacilli, bifidobacteria and propionic acid bacteria, isolated from different sources. In: Microbial pathogens and strategies for combating them: science, technology and education (A. Mendes-Vilaz ed.), Formatex. 